\title{
Intramural and subserosal echogenic foci on US in large-bowel intussusceptions: prognostic indicator for reducibility?
}

\author{
Enno Stranzinger • Michael A. DiPietro • Sai Yarram • \\ Shokoufeh Khalatbari • Peter J. Strouse
}

Received: 27 June 2008 /Revised: 16 September 2008 / Accepted: 23 September 2008 / Published online: 4 November 2008

(C) Springer-Verlag 2008

\begin{abstract}
Background In large-bowel intussusceptions, several US signs are known to indicate a lower likelihood of reducibility by enema. US can demonstrate echogenic dots or lines (foci) in the bowel wall, which might indicate an ischemic bowel.

Objective To determine the presence of echogenic intramural and subserosal foci in large-bowel intussusceptions and to evaluate the degree of correlation with reducibility.

Materials and methods Between 2001 and 2008, 74 consecutive US examinations were retrospectively evaluated by two pediatric radiologists for intramural and subserosal echogenic foci, or trapped gas, in the intussusception. The degree of correlation between the sonographic findings and reducibility was evaluated.

Results Of 73 intussusceptions examined by US, 56 (76\%) were reducible and 17 (23\%) were not reducible. Out of 10 intussusceptions with intramural gas, 11 with subserosal gas, and 14 with intramural and subserosal gas, $8(80 \%), 6$ (56\%), $9(64 \%)$, respectively, were not reducible. The presence of intramural gas or subserosal gas or both
\end{abstract}

E. Stranzinger $(\bowtie)$

Department of Diagnostic Radiology,

Inselspital Berne (University Hospital of Berne),

CH-3010 Berne, Switzerland

e-mail: enno.stranzinger@insel.ch

E. Stranzinger - M. A. DiPietro - S. Yarram - P. J. Strouse

Section of Pediatric Radiology,

University of Michigan Health System,

Ann Arbor, MI, USA

S. Khalatbari

Michigan Institute for Clinical and Health Research (MIHR),

Ann Arbor, MI, USA predicted a lower chance of reduction, but with regard to the effect of these findings together, intramural gas was the only significant predictor.

Conclusion Having intramural gas in large-bowel intussusception significantly decreases the chance of reduction.

Keywords Intussusception · US · Reducibility ·

Echogenic foci $\cdot$ Children

\section{Introduction}

Many pediatric radiologists have converted to using US to diagnose intussusception in children. In the 1980s, the use of real-time sonography was described for the diagnosis of intussusceptions with the doughnut and pseudokidney signs $[1,2]$. Yet it did not become popular in the USA until the mid- to late 1990s. Various US signs (trapped peritoneal fluid [3], absence of blood flow [4], enlarged lymph nodes [5]) predict a lower likelihood of image-guided hydrostatic or pneumatic reducibility. These US findings correlate with a lower likelihood of reduction ranging from a reduction rate of $0 \%$ for peritoneal fluid $(>14 \times 5 \mathrm{~mm})$ to a reduction rate of $40 \%$ for enlarged lymph nodes $(8.1 \mathrm{~mm}$ to $11 \mathrm{~mm})$ $[3,5]$. The generally accepted contraindications remain shock, sepsis, peritonitis and free gas [6-8].

US is able to depict echogenic dots or lines (foci) in the bowel wall that sometimes represent pneumatosis intestinalis. This finding is seen in a variety of diseases including ischemic bowel [9-13]. The presence of pneumatosis intestinalis in intussusception is likely secondary to an ischemic bowel.

We retrospectively looked for intramural echogenic foci or lines (gas) or subserosal echogenic foci on US scans in children with large-bowel intussusceptions to determine the 


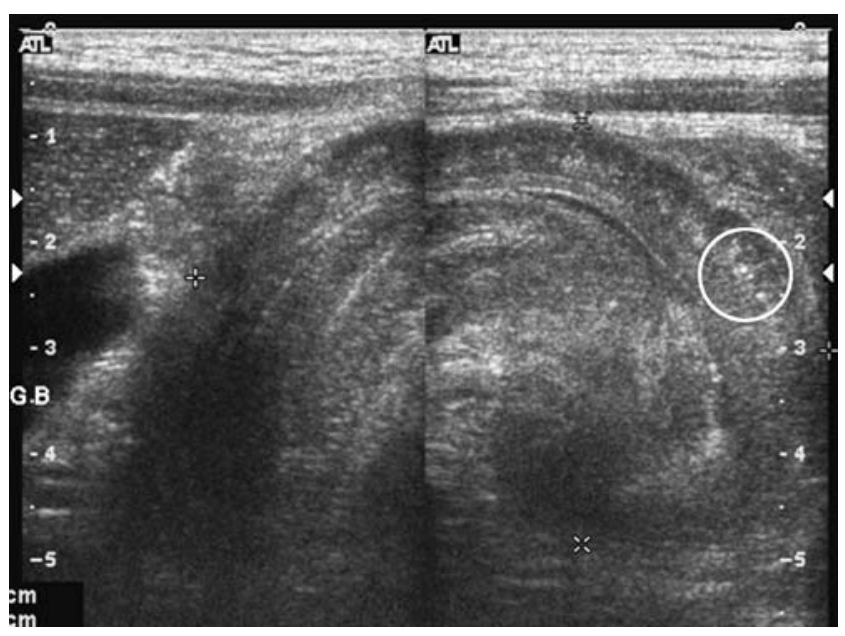

Fig. 1 Ileocecal intussusception in an 8-year-old boy. Linear transducer shows a large-bowel intussusception with intramural echogenic foci (circle)

degree of correlation with reducibility under fluoroscopic guidance (Figs. 1 and 2).

\section{Materials and methods}

We received institutional review board approval for this retrospective study. Between 2001 and 2008 there were 74 consecutive children with sonographically detected largebowel intussusception. One patient had no fluoroscopic enema study, and 73 children had subsequent fluoroscopic pneumatic (53 patients, $72 \%$ ) or hydrostatic (20 patients, $27 \%$ ) enema studies. After 2005, most enemas were performed with pneumatic reduction. Children with smallbowel intussusception and those with malignancies as pathologic lead points and follow-up studies were not included in this study. One patient with US signs of bowel ischemia went directly to surgery for intussusception reduction without having a fluoroscopic enema. This patient was excluded from the statistical analysis.
We report 23 girls (31\%) and 51 boys $(69 \%)$ between 2 months and 10 years of age (median 15.5 months).

Two pediatric radiologists examined all US images and all cine-loops for intramural gas, subserosal gas and trapped gas between bowel walls. Both readers had to agree on the findings for a scan to be positive. Cine-loops were available in 72 patients $(97 \%)$. The readers were blind to the reducibility, the clinical history, clinical findings and clinical outcome. The sonographic findings were then compared with the reducibility with hydrostatic or pneumatic enema and the surgical and pathological reports. Doppler sonography was not evaluated in this study, because it was infrequently available. Radiographs were reviewed in patients with nonreducible intussusception.

The intussusceptions were reduced with air or contrast enemas under fluoroscopic guidance. Sealing was achieved by a taped catheter and if necessary with an inflated balloon. Pressures were monitored, and were below $120 \mathrm{mmHg}$.

\section{Statistical methods}

Fisher's exact test was used for univariate analysis to evaluate the influence of each predictor on intussusception reducibility outcome. A multivariate logistic model was then fit with all significant variables to further investigate their effect on the success of the reduction. Probability $(P)$ values of 0.05 or smaller were considered significant for all hypothesis tests.

\section{Results}

In 73 consecutive children with sonographically detected large-bowel intussusception, $17(23 \%)$ intussusceptions were not reducible and $56(76 \%)$ were reducible. The patients with nonreducible intussusception were younger than those with reducible intussusception (median age 9 months and 19.5 months, respectively).
Fig. 2 Images in a 5-month-old girl who had bilious emesis, became increasingly lethargic and was passing bloody currant jelly stools. a Short-axis view of the intussusception shows echogenic lines in a thickened edematous bowel wall (circle), subserosal echogenic foci (arrow) and a fluid cap sign (asterisk). b Radiograph of the abdomen shows bubbly, gas-filled bowel in the right lower quadrant, concerning for pneumatosis (circle). No free abdominal gas or portal venous gas was noted
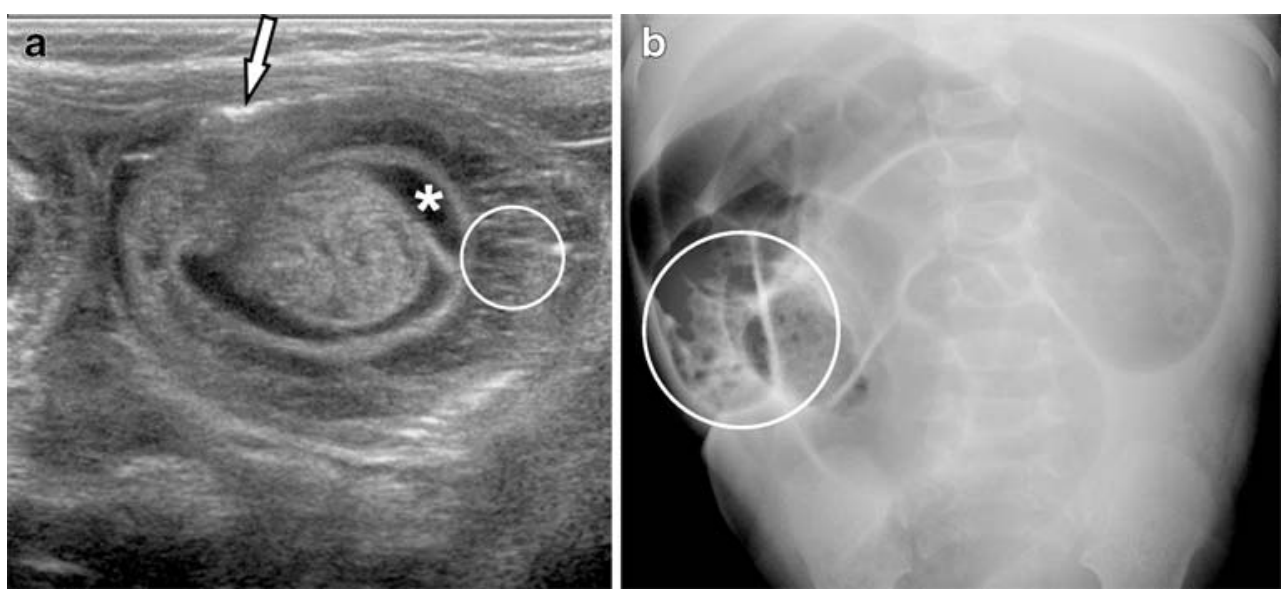
A 5-month-old girl was taken directly to the operating room without fluoroscopic reduction because the duration ( $>48 \mathrm{~h}$ ) of her symptoms and evidence of necrotic bowel on her US scan (Fig. 2) led to concern for bowel ischemia. A large area of necrotic bowel from the distal ileum up to the level of the transverse colon just distal to the hepatic flexure was found at surgery.

Among the above predictors, intramural gas $(P<0.0001)$, subserosal gas $(P=0.02)$ and intramural/subserosal gas $(P<0.0001)$ were found to be significant (Table 1$)$. The presence of intramural gas was a predictor of nonreducibility with a sensitivity of $47 \%$ and a specificity of $96 \%$. Subserosal gas had a sensitivity of $35 \%$ and a specificity of $91 \%$ while intramural/subserosal gas had a sensitivity of $53 \%$ and a specificity of $91 \%$.

Of 10 children with intramural gas, $8(80 \%)$ had nonreducible intussusception, and of 63 without intramural gas, 54 (86\%) had reducible intussusception. Of 11 children with subserosal gas, $6(56 \%)$ had nonreducible intussusception, and of 62 without subserosal gas, $51(82 \%)$ had reducible intussusception. Of 14 children with intramural and/or subserosal gas, 9 (64\%) had nonreducible intussusception, and of 59 without echogenic foci, 51 (86\%) had reducible intussusception.

Trapped gas as well as gender and age were not significantly correlated with the success of fluoroscopic intussusception reduction (all $P>0.05$ ). Multivariate analysis of the data with a model consisting of intramural, subserosal and intramural and subserosal gas as predictors showed that only intramural gas was a significant predictor of nonreducibility $(P<0.0001)$. This implies that if a patient has all three types of echogenic foci, the reducibility would be the same as if the patient had only intramural gas.

\section{Complications}

Two perforations $(2 / 73,3 \%)$ occurred during unsuccessful reduction (one with air, one with liquid contrast agent). One patient with barium enema showed a large amount of barium contamination. The intussusception was reduced during surgery and the bowel was examined during surgery and thought to be viable. There was no pathology report for this patient. A $0.4-\mathrm{cm}$ perforation was found in the transverse colon at the hepatic flexure. A 7-month-old patient with a perforation occurring during air enema had an area of $0.6 \times 0.6 \mathrm{~cm}$ with pathologically proven ischemic changes in the large bowel. Both children showed echogenic intramural foci on the US scan.

In one child, a bowel perforation occurred $6 \mathrm{~h}$ after successful pneumatic reduction. Surgery and pathology confirmed necrotic bowel in this patient (Fig. 3). This patient also had intramural and subserosal echogenic foci.

\section{Discussion}

US has been shown to be accurate with a high negative predictive value in the diagnosis of intussusception [14, 15]. Henrikson et al. [16] reported that screening US has decreased the number of enemas in a tertiary center for clinically suspected intussusception, and this has in turn reduced children's exposure to ionizing radiation. Our reduction rate of $76 \%$ seems low and the perforation rate high $(3 \%)$, but it should be noted that our hospital is a tertiary referral center with sometimes significant delay in patients' arrival, because they are often transferred from other hospitals. Several reports describe the prediction of bowel viability by US and Doppler examination in children with intussusception and correlate assorted US signs with reducibility.

According to Lim et al. [17], the absence of blood flow in the intussusceptum at examination with color Doppler sonography with a $5-$ to $10-\mathrm{MHz}$ linear array transducer suggests that gangrenous change has occurred in the intussusceptum and that vigorous reduction should not be attempted. Koumanidou et al. [5] evaluated enlarged lymph nodes in the intussusception. Enlarged lymph nodes were found in patients with a current or a recent history of gastroenteritis and correlated with a lower overall hydrostatic reduction rate. del-Pozo et al. [3] evaluated fluid seen inside the intussusception, which represented trapped peritoneal fluid. A substantial amount $(>14 \mathrm{~mm} \times 5 \mathrm{~mm})$ of trapped fluid was associated with irreducibility and bowel ischemia.

Various reports have described the US findings of ischemic bowel, in particular in children with necrotizing enterocolitis $[9,10,18]$. A variety of diagnoses are known to have intramural gas (bowel necrosis from vascular

Table 1 Significant predictors of fluoroscopic intussusception reducibility by Fisher's exact test.

\begin{tabular}{|c|c|c|c|c|}
\hline US finding & $\begin{array}{l}\text { Total no. }(\%) \text { of } \\
\text { intussusceptions }(n=73)\end{array}$ & $\begin{array}{l}\text { No. }(\%) \text { of nonreducible } \\
\text { intussusceptions }\end{array}$ & $P$ value & $\begin{array}{l}95 \% \text { confidence } \\
\text { interval }\end{array}$ \\
\hline Intramural gas & $10(14 \%)$ & $8(80 \%$ of 10$)$ & $<0.0001$ & $0.008-0.229$ \\
\hline Subserosal gas & $11(15 \%)$ & $6(56 \%$ of 11$)$ & 0.02 & $0.046-0.696$ \\
\hline Intramural and subserosal gas & $14(19 \%)$ & $9(64 \%$ of 14$)$ & $<0.0001$ & $0.023-0.327$ \\
\hline
\end{tabular}


Fig. 3 Images in a 7-week-old girl transferred from an outside hospital with intussusception. a Short-axis US image shows thickened intussusceptum with echogenic foci (circle), subserosal echogenic foci (arrow) and absent flow within the bowel wall. b Six hours after successful pneumatic reduction the left lateral decubitus view of the abdomen shows free abdominal gas (asterisk) over the liver
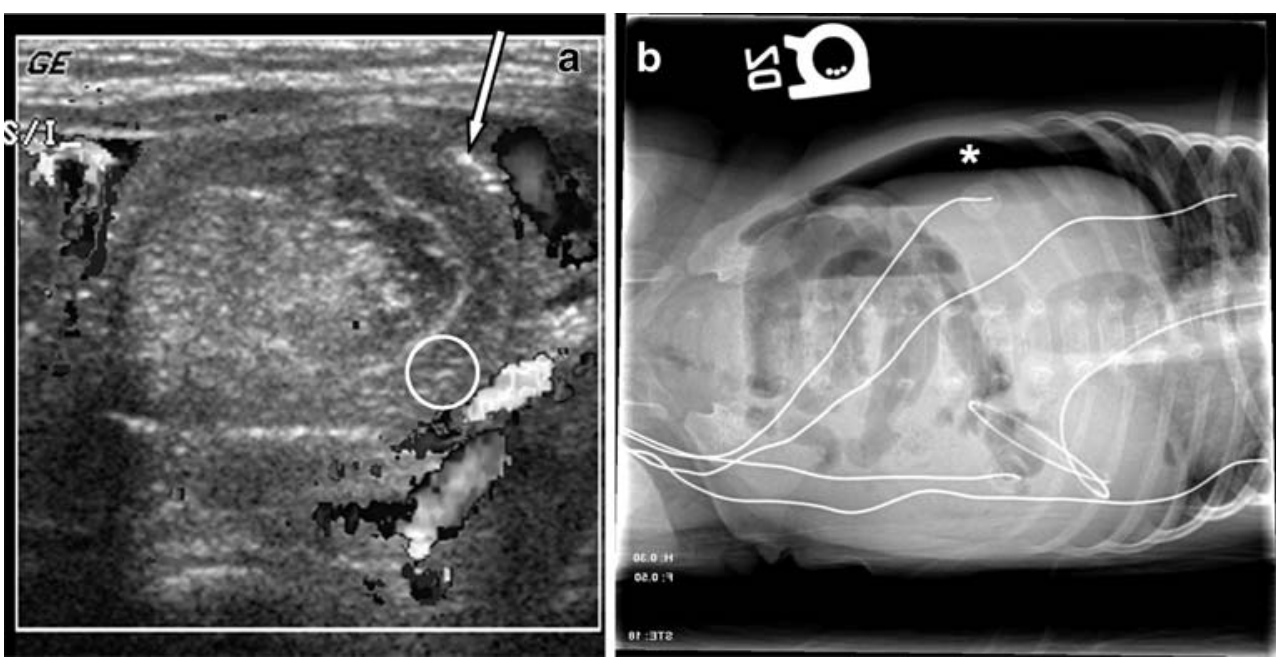

hypoperfusion, occluded or impaired venous drainage, necrotizing enterocolitis, chronic bronchitis and asthma, inflammatory bowel disease, ulcer disease and diverticulitis and collagen vascular disease) [9-13]. In the radiological evaluation of large-bowel intussusception the finding of intramural gas is likely related to ischemic bowel. Our data showed that the finding of intramural gas, subserosal gas or intramural/subserosal gas on US examination would significantly decrease the chance of successful intussusception reduction. Intramural echogenic dots or lines (gas) are a reliable sign, especially in an edematous, thickened bowel wall. Subserosal gas is much more difficult to differentiate from intraluminal gas because of the thin area of interest and the close relationship to intraluminal gas.
Artefacts

Intraluminal gas bubbles at the interface between the mucosa and the luminal contents can falsely appear to be within the gut wall itself and thereby cause the artefact "pseudopneumatosis intestinalis." The differentiation of subserosal gas and intramural gas can be influenced by technical parameters and the angle of the transducer [19]. Trapped gas and subserosal echogenic foci on a short-axis view can be difficult to differentiate and should be confirmed on a longaxis view and if possible on a cine-loop.

Another pitfall is echogenic foci that are seen on the short-axis view of the bowel as projecting into the muscularis or submucosa. When viewed on a long-axis

Fig. 4 Ileocecal intussusception in a 4-year-old boy. a Short-axis view shows echogenic mucosal fold mimicking intramural gas (arrow). b Long-axis view shows echogenic foci as trapped intraluminal gas in a mucosal fold (circle)
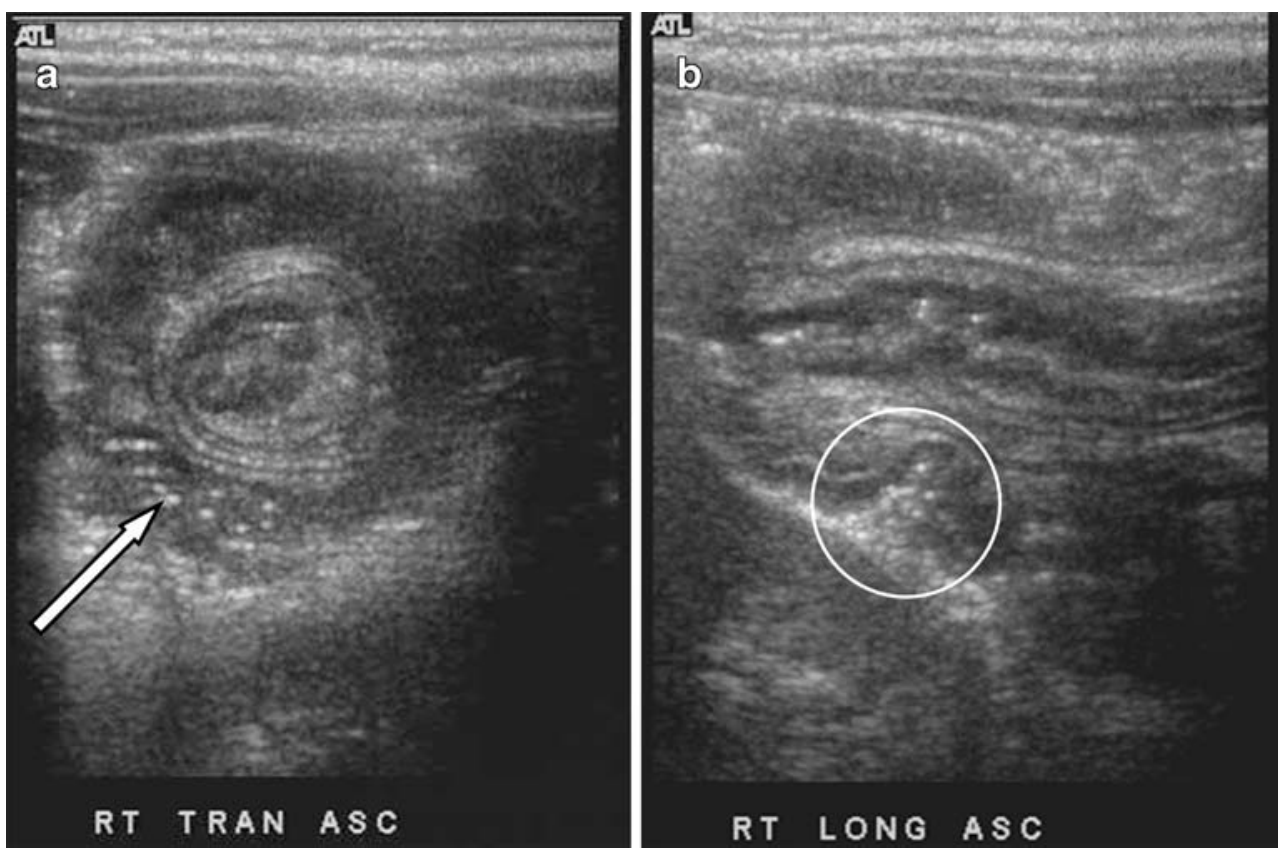
view of the bowel the foci are noted to be within an intraluminal mucosal fold (Fig. 4).

Perforations during enema examinations are rare, but can occur and can have catastrophic consequences [6]. In one child the radiologist was able to reduce the intussusception, but the patient presented hours later with a perforation. Pathology proved necrotic bowel, and the US scan showed intramural gas (Fig. 3). This case shows that even necrotic bowel might be reducible with pneumatic or hydrostatic enema, but complications might occur later.

Another child went directly to surgery because of US findings of intramural gas and absence of Doppler flow in the bowel wall. Necrotic bowel was also found in this patient intraoperatively (Fig. 2). Plain films are recommended to rule out free abdominal gas but can also show the bubbly appearance of intramural gas in necrotic bowel (Fig. 3).

This retrospective study showed that the presence of intramural gas, subserosal gas or both in patients with largebowel intussusception can significantly decrease the chance of successful intussusception reduction. In patients without signs of pneumatosis, there is more than a $90 \%$ chance of reduction.

\section{Study limitations}

The retrospective nature of the study is a limitation. Not all US examinations were standardized for the specific question of ischemic bowel and not all included Doppler images. Some US examinations were only performed with curved abdominal transducers, without linear transducers.

\section{Conclusion}

Intramural or subserosal gas (pneumatosis intestinalis) in children with large-bowel intussusception suggests intestinal ischemia with pneumatosis or advanced changes of intussusception. These findings are associated with a higher likelihood of nonreducibility and thereby an increased risk of bowel perforation. A prospective study might be necessary to confirm the significance of the various US signs for nonreducibility in large-bowel intussusception.

Acknowledgement The project was supported by a grant from the National Center for Research Resources (no. UL1RR024986). The content is solely the responsibility of the authors and does not necessarily represent the official views of NCRR or the National Institutes of Health.

\section{References}

1. Bowerman RA, Silver TM, Jaffe MH (1982) Real-time ultrasound diagnosis of intussusception in children. Radiology 143:527-529

2. Swischuk LE, Hayden CK, Boulden T (1985) Intussusception: indications for ultrasonography and an explanation of the doughnut and pseudokidney signs. Pediatr Radiol 15:388-391

3. del-Pozo G, Gonzalez-Spinola J, Gomez-Anson B et al (1996) Intussusception: trapped peritoneal fluid detected with US relationship to reducibility and ischemia. Radiology 201:379-383

4. Hanquinet S, Anooshiravani M, Vunda A et al (1998) Reliability of color Doppler and power Doppler sonography in the evaluation of intussuscepted bowel viability. Pediatr Surg Int 13:360-362

5. Koumanidou C, Vakaki M, Pitsoulakis G et al (2002) Sonographic detection of lymph nodes in the intussusception of infants and young children: clinical evaluation and hydrostatic reduction. AJR $178: 445-450$

6. Berlin L (1998) Reducing the intussuscepted colon. AJR 170:1161-1163

7. Daneman A, Navarro O (2004) Intussusception. Part 2: an update on the evolution of management. Pediatr Radiol 34:97-108

8. del-Pozo G, Albillos JC, Tejedor D et al (1999) Intussusception in children: current concepts in diagnosis and enema reduction. Radiographics 19:299-319

9. Bloom RA, Craciun E, Lebensart PD et al (1992) The ultrasound appearances of intramural bowel gas: the bright ring appearance and the effervescent bowel. A report of three cases. Br J Radiol 65:585-588

10. Kim WY, Kim IO, Kim WS et al (2007) Sonographic findings in a model of ischemia-induced necrotizing enterocolitis with pathological correlations. Invest Radiol 42:312-318

11. Oktar SO, Yucel C, Erbas G et al (2006) Use of twinkling artifact in sonographic detection of intestinal pneumatosis. Abdom Imaging 31:293-296

12. Romano S, Lassandro F, Scaglione $M$ et al (2006) Ischemia and infarction of the small bowel and colon: spectrum of imaging findings. Abdom Imaging 31:277-292

13. St Peter SD, Abbas MA, Kelly KA (2003) The spectrum of pneumatosis intestinalis. Arch Surg 138:68-75

14. Pracros JP, Tran-Minh VA, Morin de Finfe CH et al (1987) Acute intestinal intussusception in children. Contribution of ultrasonography (145 cases). Ann Radiol (Paris) 30:525-530

15. Verschelden P, Filiatrault D, Garel L et al (1992) Intussusception in children: reliability of US in diagnosis - a prospective study. Radiology 184:741-744

16. Henrikson S, Blane CE, Koujok K et al (2003) The effect of screening sonography on the positive rate of enemas for intussusception. Pediatr Radiol 33:190-193

17. Lim HK, Bae SH, Lee KH et al (1994) Assessment of reducibility of ileocolic intussusception in children: usefulness of color Doppler sonography. Radiology 191:781-785

18. Faingold R, Daneman A, Tomlinson G et al (2005) Necrotizing enterocolitis: assessment of bowel viability with color Doppler US. Radiology 235:587-594

19. Wilson SR, Burns PN, Wilkinson LM et al (1999) Gas at abdominal US: appearance, relevance, and analysis of artifacts. Radiology 210:113-123 